\title{
ON RULED SURFACES WHOSE FLECNODE CURVE INTERSECTS EVERY GENERATOR IN TWO COINCIDENT POINTS*
}

BY

\section{E. J. WILCZYNSKI $†$}

The formulæ and the theorems developed in my recent paper Studies in the general theory of ruled surfaces are not directly applicable to the case when $\theta_{4}=0, i$. e., when the flecnode curve intersects every generator in two coincident points. The general notions, employed in that paper, $\ddagger$ may however be applied to this special case as well, and give rise to a number of interesting and important considerations.

\section{§1. The covariant $C_{3}$.}

The interpretation of the covariant $C_{3}$ given in Covariants and Studies is complete for the case $\theta_{4} \neq 0$. It breaks down absolutely for $\theta_{4}=0$.

This covariant is

$$
C_{3}=\alpha z-\beta y
$$

where

$$
\begin{aligned}
& \alpha=2\left(u_{11}-u_{22}\right) \rho+4 u_{12} \sigma+\frac{1}{2}\left(v_{11}-v_{22}\right) y+v_{12} z, \\
& \beta=4 u_{21} \rho-2\left(u_{11}-u_{22}\right) \sigma+v_{21} y-\frac{1}{2}\left(v_{11}-v_{22}\right) z . \S
\end{aligned}
$$

We have

$$
\theta_{4}=\left(u_{11}-u_{22}\right)^{2}+4 u_{12} u_{21}=0 .
$$

Let us assume that the curve $C_{y}$ is the flecnode curve, so that $u_{12}=0$. We shall then have also $u_{11}-u_{22}=0$. Further we may assume $p_{11}=p_{22}=0$.

We have therefore

whence

$$
u_{12}=u_{11}-u_{22}=0, \quad p_{11}=p_{22}=0,
$$

$$
\alpha=p_{12} u_{21} y, \quad \beta=4 u_{21} \rho+v_{21} y-p_{12} u_{21} z .
$$

* Presented to the Society December 29, 1903. Received for publication November 15, 1903. $\dagger$ Of the Carnegie Institution of Washington.

$\ddagger$ Hereafter referred to as Studies.

$\S$ Covariants, p. 448. 
If a transformation of the independent variable be made by putting $\xi=\xi_{(x)}$, we find that for the new system of differential equations

$$
\bar{v}_{21}=\frac{1}{\left(\xi^{\prime}\right)^{3}}\left(v_{21}-4 u_{21} \eta\right), \quad \eta=\frac{\xi^{\prime \prime}}{\xi^{\prime}} \cdot *
$$

Therefore if $u_{21} \neq 0$, i. e., if $S$ is not a quadric, we can always choose $\eta$ in just one way so as to make $\bar{v}_{21}=0$. We obtain therefore a perfectly definite surface of the congruence $\Gamma$, which we will call its principal surface, and which we shall characterize geometrically farther on.

Let us assume that the variable $x$ has already been chosen in such a way that $S^{\prime}$, the derivative of $S$ with respect to $x$, shall coincide with the principal surface of $\Gamma$. Then $v_{21}=0$, and (3) becomes

where

$$
\alpha=p_{12} u_{21} y, \quad \beta=4 u_{21} \rho-p_{12} u_{21} z=u_{21} \bar{\beta},
$$

$$
\bar{\beta}=4 \rho-p_{12} z .
$$

We have further in general (Covariants, p. 430),

whence

$$
\rho=2 y^{\prime}+p_{11} y+p_{12} z,
$$

$$
2 y^{\prime}=\rho-p_{12} z .
$$

The point, whose coördinates are $y_{1}^{\prime}, \ldots, y_{4}^{\prime}$, is therefore obviously the intersection of the tangent to the flecnode curve with the line $P_{z} P_{\rho}$. The point $P_{\beta}$ whose coördinates are given by $\beta_{1} \ldots \beta_{4}$ is also on the line $P_{z} P_{\rho}$, and the cross ratio of the four points $P_{\rho} P_{\beta} P_{z} P_{y^{\prime}}$ is $\frac{1}{4}$. The point $P_{a}$ obviously coincides with $P_{y}$.

The ruled surface which the covariant $C_{3}$ adjoins to $S$ may therefore be defined as follows. In the plane tangent to $S$ at its flecnode $P_{y}$, construct a line passing through $P_{y}$ such that it, together with the generator, the flecnode tangent and the tangent of the flecnode curve shall constitute a plane pencil whose anharmonic ratio is $\frac{1}{4}$. The locus of these lines is the required ruled surface. Moreover the points of any generator of this surface are, by means of the covariant $C_{3}$ put into a one-to-one correspondence with those of $g$. The lines joining corresponding points pass through $P_{\rho}$, that point of the principal surface of the congruence $\Gamma$ which corresponds to $P_{y}$,

It only remains to give a characteristic geometric property of the principal surface of $\Gamma$. For this purpose let us assume, in addition to our previous hypotheses, that $C_{z}$ is an asymptotic curve on $S$, i. e., let $p_{21}=0$. Then (Congruence, p. 188).

* Congruence, p. 187. 
and

$$
\begin{aligned}
& 2 \sigma^{\prime}=u_{21} y+u_{11} z, \\
& 2 \sigma^{\prime \prime}=u_{21}^{\prime} y+\left(u_{11}^{\prime}-\frac{1}{2} u_{21} p_{12}\right) z+\frac{1}{2}\left(u_{21} \rho+u_{11} \sigma\right),
\end{aligned}
$$

$$
v_{21}=2 u_{21}^{\prime} \text {. }
$$

The first equation shows that the tangent to the curve $C_{\sigma}$ at $P_{\sigma}$ intersects the generator $g$ of $S$. Denote this point of intersection by $P_{\sigma^{\prime}}$. The locus of the point $P_{\sigma^{\prime}}$ is therefore a curve on $S, C_{\sigma^{\prime}}$. Its tangent at $P_{\sigma^{\prime}}$ is obtained by joining $P_{\sigma^{\prime}}$ to the point $P_{\sigma^{\prime \prime}}$ defined by the second equation (4). But $P_{\sigma^{\prime \prime}}$ is in the plane $P_{z} P_{\rho} P_{\sigma}$ if and only if $v_{21}=0$. Put

$$
\tau=u_{21} \rho+u_{11} \sigma
$$

then $P_{\tau}$ is a point on $P_{\rho} P_{\sigma}$ such that the line joining it to $P_{\sigma^{\prime}}$ is a generator of the hyperboloid $H$ osculating $S$ along $g$. We see that the tangent to $C_{\sigma}$, intersects $P_{z} P_{\tau}$ if and only if $v_{21}=0$.

The principal surface of the congruence $\Gamma$ is therefore defined by the following theorem :

We draw upon the ruled surface $S$ any asymptotic line $C_{z}$, and upon any surface $S^{\prime}$ of the congruence $\Gamma$ the curve $C_{\sigma}$ which corresponds to it, $P_{z}$ and $P_{\sigma}$ being corresponding points of the two curves. The tangent to $C_{\sigma}$ at $P_{\sigma}$ always intersects the generator $g$ of $S$, which passes through $P_{z}$, in a certain point $P_{\sigma^{\prime}}$, whose locus gives a curve $C_{\sigma^{\prime}}$ upon $S . \quad U$ pon $S^{\prime}$ a point $P_{\tau}$ is constructed such that the line $P_{\tau} P_{\sigma^{\prime}}$ shall be a generator of the hyperboloid osculating $S$ along $g$. The tangent to $C_{\sigma^{\prime}}$ at $P_{\sigma^{\prime}}$ intersects the line $P_{z} P_{\tau}$ if and only if the surface $S^{\prime}$ is the principal surface of the congruence.

\section{$\S 2$. The derivative conic.}

In the general case where $\theta_{4} \neq 0$, the hyperboloid $H$ osculating $S$ along $g$ and the hyperboloid $H^{\prime}$ osculationg $S^{\prime}$ along $g^{\prime}$ intersect along $g^{\prime}$ and a space cubic which we have called the derivative cubic. Moreover this cubic does not degenerate unless either $S$ has a straight line directrix or $S^{\prime}$ is developable.

In the present case however, $H$ and $H^{\prime}$ have besides $g^{\prime}$ the straight line $f$ in common, i. e., the flecnode tangent passing through $P_{y}$. The rest of their intersection is therefore a conic which we shall call the derivative conic.

$\mathrm{We}$ proceed to prove these statements and to derive the equations of the derivative conic. Taking as tetrahedron of reference the tetrahedron $P_{y} P_{z} P_{p} P_{\sigma}$, the equation of $H$ is

and that of $H^{\prime}$

$$
x_{1} x_{4}-x_{2} x_{3}=0,
$$

$$
\begin{aligned}
& \left(u_{22} x_{1}-u_{21} x_{2}\right)\left[J x_{4}-\left(\lambda_{12} u_{22}-\lambda_{22} u_{12}\right) x_{1}+\left(\lambda_{12} u_{21}-\lambda_{22} u_{11}\right) x_{2}\right] \\
& \quad+\left(u_{12} x_{1}-u_{11} x_{2}\right)\left[J x_{3}-\left(\lambda_{11} u_{22}-\lambda_{21} u_{12}\right) x_{1}+\left(\lambda_{11} u_{21}-\lambda_{21} u_{11}\right) x_{2}\right]=0,
\end{aligned}
$$

as we have shown in Studies, equations (1) and (3). 
In our case we may put

whence

$$
u_{12}=u_{11}-u_{22}=0, \quad J=u_{11}^{2}
$$

$$
v_{11}-v_{22}=2 p_{12} u_{21}, \quad v_{12}=0, \quad v_{21}=2 u_{21}^{\prime}-\left(p_{11}-p_{22}\right) u_{21}
$$

and

$$
\begin{array}{ll}
2 J \lambda_{11}=-u_{11} v_{11}, & 2 J \lambda_{12}=0, \\
2 J \lambda_{21}=u_{21} v_{22}-u_{11} v_{21}, & 2 J \lambda_{22}=-u_{11} v_{22} .
\end{array}
$$

We find, therefore, for $H^{\prime}$ the equation

$\left(u_{11} x_{1}-u_{21} x_{2}\right)\left[J x_{4}-\lambda_{22} u_{11} x_{2}\right]-u_{11} x_{2}\left[J x_{3}-\lambda_{11} u_{11} x_{1}+\left(\lambda_{11} u_{21}-\lambda_{21} u_{11}\right) x_{2}\right]=0$, or

(7) $J u_{11}\left(x_{1} x_{4}-x_{2} x_{3}\right)+u_{11}^{2}\left(\lambda_{11}-\lambda_{22}\right) x_{1} x_{2}-J u_{21} x_{2} x_{4}+\left[u_{11}^{2} \lambda_{21}-u_{11} u_{21}\left(\lambda_{11}-\lambda_{22}\right)\right] x_{2}^{2}=0$, while the equation of $H$ is

$$
x_{1} x_{4}-x_{2} x_{3}=0 \text {. }
$$

Both equations are satisfied by $x_{1}=x_{2}=0$, as well as by $x_{2}=x_{4}=0$ which proves that $g^{\prime}$ and $f$ are lines upon both of these hyperboloids. They must therefore have also a conic in common, whose plane must, according to $(7)$, have the equation

$$
u_{11}\left(\lambda_{11}-\lambda_{22}\right) x_{1}-u_{11} u_{21} x_{4}+\left[u_{11} \lambda_{21}-u_{21}\left(\lambda_{11}-\lambda_{22}\right)\right] x_{2}=0 .
$$

If we put for abbreviation

$$
A=u_{11}\left(\lambda_{11}-\lambda_{2:}\right)
$$$$
B=-u_{11} \lambda_{21}+u_{21}\left(\lambda_{11}-\lambda_{22}\right),
$$$$
C=u_{11} u_{21}
$$

whence

$$
u_{11} A+p_{12} C=0,
$$

we have therefore, as the equations of the derivative conic

$$
A x_{1}-B x_{2}-C x_{4}=0, \quad x_{1} x_{4}-x_{2} x_{3}=0 .
$$

We can also express the coördinates of any point on the conic in terms of a parameter $t$. Any point on the hyperboloid $H$ can be represented in the form

$$
x_{1}=u t, \quad x_{2}=t, \quad x_{3}=u, \quad x_{4}=1 .
$$

This point is, moreover, a point of the conic if the condition

is satisfied, whence

$$
A u t-B t-C=0
$$

$$
u=\frac{C+B t}{A t} .
$$


If we substitute into the above equations for $x_{1} \cdots x_{4}$ and multiply by $A t$, we find

$$
x_{1}=t(C+B t), \quad x_{2}=A t^{2}, \quad x_{3}=C+B t, \quad x_{4}=A t
$$

as the parametric equations of the conic, or in homogeneous form

$$
x_{1}=t_{1} \psi, \quad x_{2}=A t_{1}^{2}, \quad x_{3}=t_{2} \psi, \quad x_{4}=A t_{1} t_{2},
$$

$$
\psi=B t_{1}+C t_{2} \text {. }
$$

The conic of course always passes through $P_{\rho}$. The first question which we naturally ask is this: when does the conic degenerate into a pair of lines? Clearly this can only happen if the plane

$$
A x_{1}-B x_{2}-C x_{4}=0
$$

intersects the hyperboloid $H$ in a pair of lines, i. e., if it is tangent to $H$. Moreover since this plane contains $P_{\rho}$, it must in that case contain at least one of the two generators of $H$ which pass through $P_{\rho}$. If it contains that one which passes also through $P_{\sigma}$ we must have $C=0$, i. e., since $u_{21} \neq 0$ ( $S$ not being a quadric), $u_{11}=0 . \quad S^{\prime}$ must therefore be a developable. If however this plane contains the other generator through $P_{\rho}$, namely that one which passes through $P_{y}$, we must have $A=0$, which gives either $u_{11}=0$ as before or else $p_{12}=0$, in which case the flecnode curve $C_{y}$ would be a straight line.

Therefore, the derivative conics degenerate if and only if the surface $S$ has a straight line directrix, or else if the derivative of $S$ with respect to $x$ is one of the developable surfaces of the congruence $\Gamma$.

By an investigation similar to that in Studies, pp. 230-232, we obtain the further result :

Two consecutive derivative conics never intersect unless they degenerate.

§3. The developable surface generated by the plane of the derivative conic.

As $x$ changes, the plane of the conic $C_{x}$ envelops a developable surface, the equations of whose generator we shall now proceed to determine.

Let us form $\partial \phi / \partial x$ under the assumption that $t_{1}: t_{2}$ is independent of $x$. Then

$$
\phi+\frac{\partial \phi}{\partial x} \delta x
$$

will represent any point on the derivative conic $C_{x+\delta x}$ belonging to the argument $x+\delta x$, where $\delta x$ is an infinitesimal. The plane of this conic will be determined by any three points upon it. We have (again assuming $p_{21}=0$ ), 


$$
\begin{aligned}
\frac{\partial \phi}{\partial x}=y[ & \left.t_{1}\left(B^{\prime} t_{1}+C^{\prime} t_{2}\right)+\frac{1}{2}\left(B t_{1}+C t_{2}\right) u_{11} t_{2}+\frac{1}{2} A t_{1} t_{2} u_{21}\right] \\
& +z\left[A^{\prime} t_{1}^{2}-\frac{1}{2}\left(B t_{1}+C t_{2}\right) p_{12} t_{1}+\frac{1}{2} A t_{1} t_{2} u_{11}\right] \\
& +\rho\left[t_{2}\left(B^{\prime} t_{1}+C^{\prime} t_{2}\right)+\frac{1}{2}\left(B t_{1}+C t_{2}\right) t_{1}\right] \\
& +\sigma\left[A^{\prime} t_{1} t_{2}-\frac{1}{2}\left(B t_{1}+C t_{2}\right) p_{12} t_{2}+\frac{1}{2} A t_{1}^{2}\right] .
\end{aligned}
$$

We can obtain three points of the conic $C_{x+\delta x}$ by putting $t_{1}=0, t_{2}=1$; $t_{1}=1, t_{2}=0 ; t_{1}=+C, t_{2}=-B$, which last set of values corresponds to $\psi=0$. Therefore the equation of the plane of $C_{x+\delta x}$ is

$\left|\begin{array}{cccc}x_{1} & x_{2} & x_{3} & x_{4} \\ C u_{11} \delta x & 0 & 2 C+2 C^{\prime} \delta x & -C p_{12} \delta x \\ 2 B+2 B^{\prime} \delta x & 2 A+\left(2 A^{\prime}-B p_{12}\right) \delta x & B \delta x & A \delta x \\ {\left[2 C\left(B^{\prime} C-B C^{\prime}\right)\right.} & 2 A C^{2}+\left(2 A^{\prime} C^{2}\right. & -2 B\left(B^{\prime} C\right. & -2 A B C+\left(A C^{2}\right. \\ \left.-A B C u_{21}\right] \delta x & \left.-A B C u_{11}\right) \delta x & \left.-B C^{\prime}\right) \delta x & \left.-2 A^{\prime} B C\right) \delta x\end{array}\right|=0$.

If this determinant be developed, retaining of course only the terms of the first order in $\delta x$, we find that the planes of $C_{x}$ and of $C_{x+\delta x}$ intersect along the line

where

$$
\begin{aligned}
A x_{1}-B x_{2}-C x_{4} & =0, \\
A D x_{1}-E x_{2}-C F x_{4} & =0,
\end{aligned}
$$

$$
\begin{aligned}
& D=4 A^{\prime} C+2 A C^{\prime}-B C p_{12} \\
& E=2 B C A^{\prime}+2 A C B^{\prime}+2 A B C^{\prime}-A C^{2} \\
& F=4 A C^{\prime}+2 A^{\prime} C-A B u_{11}+A^{2} u_{21}=4 A C^{\prime}+2 A^{\prime} C+A u_{11}^{2} \lambda_{21} .
\end{aligned}
$$

Equations (15) are therefore the equations of the generators of the developable surface.

This proof would not be valid if either $C$ or $B$ were zero. For then the third of the three points of the conic $C_{x+\delta x}$ which we have used to determine its plane would coincide with one of the other two. If $C=0$ the conic degenerates. Since however we might in the case $B=0$ choose three other points of $C_{x+\delta x}$, as we might also do in the general case, the result will obviously be obtained from the general case by substituting $B=0$.

We see from (15) that the generator of the developable surface passes through $P_{\rho}$ and that it intersects the plane $x_{3}=0$, in the point $P_{\chi}$ or

$$
[C(E-B F), A C(D-F), 0, A(E-B D)],
$$

which we may therefore represent by the expression 


$$
\chi=C(E-B F) y+A C(D-F) z+A(E-B D) \sigma .
$$

Since the surface generated by this line joining $P_{\rho}$ to $P_{x}$ is developable, it must be possible to find four functions $\alpha, \beta, \gamma, \delta$ of $x$ such that

Now we have

and we find

$$
\alpha \rho+\beta \chi+\gamma \rho^{\prime}+\delta \chi^{\prime}=0 \text {. }
$$

$$
\rho^{\prime}=\frac{1}{2}\left(u_{11} y-p_{12} \sigma\right),
$$

where

$$
\chi^{\prime}=G y+H z+M \rho+N \sigma,
$$

$$
\begin{aligned}
& G=C\left(E^{\prime}-B F^{\prime}-B^{\prime} F^{\prime}\right)+C^{\prime}\left(E-B F^{\prime}\right)+\frac{1}{2} u_{21} A(E-B D), \\
& H=\left(A C^{\prime}+A^{\prime} C^{\prime}\right)\left(D-F^{\prime}\right)+A C\left(D^{\prime}-F^{\prime}\right)-\frac{1}{2} p_{12} C(E-B F) \\
& \quad+\frac{1}{2} u_{11} A(E-B D), \\
& M=\frac{1}{2} C(E-B F), \\
& N=A^{\prime}(E-B D)+A\left(E^{\prime}-B D^{\prime}-B^{\prime} D\right)+\frac{1}{2} A C(D-F) .
\end{aligned}
$$

If we substitute these values of $\chi^{\prime}$ and $\rho^{\prime}$ and also the expression (17) for $\chi$ into (18), we find that $\alpha, \beta, \gamma, \delta$ must satisfy the equations

$$
\begin{aligned}
C(E-B F) \beta+\frac{1}{2} u_{11} \gamma+G \delta & =0, \\
A C(D-F) \beta+H \delta & =0, \\
A(E-B D) \beta-\frac{1}{2} p_{12} \gamma+N \delta & =0, \\
\alpha+M \delta & =0 .
\end{aligned}
$$

Therefore the determinant of the first three equations, which expanded becomes

$$
\frac{1}{2} p_{12} C\left(D-F^{\prime}\right)[N C-G A+H B] \text {, }
$$

must vanish identically; i. e., since the other factors do not vanish identically, we must have

$$
N C-G A+H B=0 .
$$

We may also verify (23) directly. For we find from (20),

$$
\begin{aligned}
N C- & G A+H B=F\left(A C B^{\prime}-\frac{1}{2} A C^{2}-B A^{\prime} C+\frac{1}{2} B^{2} C p_{12}\right) \\
& -D\left(A C B^{\prime}-\frac{1}{2} A C^{2}-B A C^{\prime}-\frac{1}{2} A^{2} B u_{21}+\frac{1}{2} A B^{2} u_{11}\right) \\
& +E\left(A^{\prime} C-A C^{\prime}-\frac{1}{2} A^{2} u_{21}-\frac{1}{2} C B p_{12}+\frac{1}{2} A B u_{11}\right) \\
& =\frac{1}{2}\left[F^{\prime}(E-B D)-D\left(E-B F^{\prime}\right)+E(D-F)\right]=0 .
\end{aligned}
$$

We can now determine the edge of regression of the developable surface. If $\gamma \rho+\delta \chi$ is a point on this curve, its tangent constructed at that point must coincide with the generator of the developable, i. e., 
or

$$
\gamma^{\prime} \rho+\delta^{\prime} \chi+\gamma \rho^{\prime}+\delta \chi^{\prime}=\lambda \chi+\mu \rho
$$

$$
\left(\gamma^{\prime}-\mu\right) \rho+\left(\delta^{\prime}-\lambda\right) \chi+\gamma \rho^{\prime}+\delta \chi^{\prime}=0,
$$

which is identical with $(18)$ if we put there

$$
\alpha=\gamma^{\prime}-\mu, \quad \beta=\delta^{\prime}-\lambda .
$$

But on account of (23) we can determine $\alpha, \beta, \gamma, \delta$ so as to satisfy (18); we can therefore determine $\lambda, \mu, \gamma, \delta$ so as to satisfy (24). Moreover we find

$$
\gamma: \delta=-2 G A\left(D-F^{\prime}\right)+2 H\left(E-B F^{\prime}\right): u_{11} A\left(D-F^{\prime}\right) .
$$

Therefore the edge of regression of the developable is given by the expression

$$
\begin{aligned}
\kappa=[ & -2 G A(D-F)+2 H(E-B F)] \rho \\
& +u_{11} A(D-F)[C(E-B F) y+A C(D-F) z+A(E-B D) \sigma] .
\end{aligned}
$$

We see from (15) that the generator of the developable surface coincides with one of the generators of $H$ which passes through $P_{\rho}$, only if either $A$ or $C$ vanishes, i. e., either if $S^{\prime}$ is developable or if $S$ has a straight line directrix, in which cases the derivative conic degenerates. The generator of the developable is tangent to $H$ at $P_{\rho}$, neglecting the cases just mentioned only if $D-F=0$. As (26) shows, the cuspidal edge of the developable then coincides with $C_{\rho}$. If the expressions for $A, B, C$ be substituted into the condition $D-F=0$, or

it becomes

$$
2\left(A^{\prime} C-A C^{\prime}\right)-B C p_{12}+A B u_{11}-A^{2} u_{21}=0,
$$

which gives on integration

$$
4 \frac{u_{11}^{\prime}}{u_{11}}-2 \frac{p_{12}^{\prime}}{p_{12}}-2 \frac{u_{21}^{\prime}}{u_{21}}=0
$$

$$
\frac{u_{11}^{4}}{p_{12}^{2} u_{21}^{2}}=\text { const. }
$$

If $E-B F=0, P_{\kappa}$ lies in the plane $P_{\rho} P_{z} P_{\sigma}$, and if $E-B D=0$ in the plane $P_{y} P_{z} P_{\rho}$.

It will clearly be possible to characterize special classes of ruled surfaces (for $\theta_{4}=0$ ) by special properties of the developable surfaces here considered. As we are here dealing with the general theory of such surfaces only, we will not go into such details at present.

We will also merely mention the fact that the relation of $S$ to its flecnode surface $F$ is especially close in this case $\left(\theta_{4}=0\right)$. In fact $S$ is also the flecnode surface of $F$. Moreover, the same hyperboloid $I I$ which osculates $S$ along $g$, also osculates $F$ along the corresponding generators $f$ of $F$. The con- 
gruence $\Gamma^{\prime}$ which belongs to the surface $F$ in the same way as $\Gamma$ does to $S$, is therefore made up of the generators of the second set on the osculating hyperboloids of $S$, those of the first set constituting the lines of the congruence $\Gamma$. All of these remarks follow easily from the equations of the flecnode surface, which, under the assumptions $p_{11}=p_{22}=p_{21}=0$, assume the form

$$
\begin{array}{r}
y^{\prime \prime}-2 \frac{q_{12}}{p_{12}} y^{\prime}-\rho^{\prime}-q_{11} y+\frac{q_{12}}{p_{12}} \rho=0, \\
\rho^{\prime \prime}+{ }^{4} q_{11} y^{\prime}-2 \frac{q_{12}}{p_{12}} \rho^{\prime}+\left[2 q_{11}^{\prime}-p_{12} q_{21}-4 q_{11} \frac{q_{12}}{p_{12}}\right] y-q_{11} \rho=0 .
\end{array}
$$

Moreover, since $S^{\prime}$ is a developable surface of the congruence $\Gamma$ if $q_{11}=0$, and since (28) shows that $C_{\rho}$ is then an asymptotic curve on $F$, we see that the developable surfaces of the congruence intersect its focal surface $F$ along asymptotic lines, as it should according to the general theory of congruences.

PaRIS, October 30, 1903. 